\title{
Global Local Structural Optimization of Transportation Aircraft Wings
}

\author{
Pier Davide Ciampa* and Björn Nagel ${ }^{\dagger}$ \\ German Aerospace Center DLR, Hamburg, 21079, Germany \\ Michel van Tooren ${ }^{\ddagger}$ \\ Delft University of Technology, Delft, 2625 HH, The Netherlands
}

\begin{abstract}
The study presents a multilevel optimization methodology for the preliminary structural design of transportation aircraft wings. A global level is defined by taking into account the primary wing structural components (i.e., ribs, spars and skin) which are explicitly modeled by shell layered finite elements. Wing substructures such as stringers are implicitly represented by an equivalent formulation of the structural properties. The global level is analyzed and optimized for minimum mass under element stress constraints. Selected wing skin panels are extracted from the global wing and further remodeled with detailed stringers. Boundary conditions are transferred from the finite element (FE) global level solution to the FE detailed stiffened panel models. A finite element analysis is performed on the selected local level panels, which are mass optimized under additional stability constraints, providing a new optimal skin-stringer layout. The global model is then updated with the local level optimum results, and a number of iterative global-local optimization loops are executed. In the current study the DLR in-house tools are used for the structural modeling and sizing of the wing global level, and a new stiffened panel generator is introduced for the local level modeling. A local optimization module which includes instability failure criteria is implemented to redesign the stiffened panels for minimum mass. The global and the local levels communicate through a framework developed to assist an automated and flexible multilevel optimization, and to minimize the time consuming activities required to generate detailed finite element models. The methodology is tested and demonstrated using a transportation DLR aircraft wing geometry as global level, and a variable number of upper skin blade stiffened panels remodeled in detail as local level.
\end{abstract}

\section{Introduction}

H IGHER order effects play an increasingly dominant role in the preliminary design of transportation air-

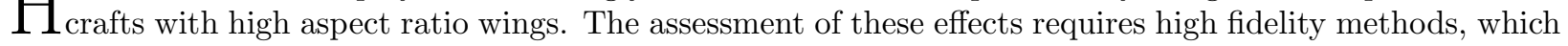
are based on explicit detailed models not always available in the preliminary stages of the design. Due to the large number of elements and substructures to be modeled, the wing structural design process can be complex, and the number of design variables involved may be unfeasible when more concepts need to be evaluated. The search for an optimum solution may simply become too time consuming, in spite of the computational power nowadays available. Thus the pre-design of aircraft wing structures relies on finite element models of significantly reduced complexity in which only spar and rib components are explicitly modeled, whereas the wing skin-stringers stiffened panels are implicitly represented by stiffness-equivalent elements. This conventional procedure enables a fast solution technique for large problems, but does not provide enough information for the sizing of the stiffened panel substructures such as the stringers dimensioning. Decomposing the main problem into subproblems of smaller scale offers a way to increase the analysis fidelity level of details, with relief of the computational efforts respect to a fully detailed model. ${ }^{1}$ Hence

*Researcher, Air Transportation Concepts and Technology Assessment, Blohmstr.18 Hamburg.

${ }^{\dagger}$ Head Aircraft Design, Air Transportation Concepts and Technology Assessment, Blohmstr.18 Hamburg. Member AIAA.

¥Full Professor, Faculty of Aerospace Engineering, Kluyverweg 1 Delft. AIAA MDO TC member. 
the global-local optimization appears to be a suitable approach to enhance the fidelity of the analysis to be performed, and to enable a fast solution of large scale problems at the same time. Bringing more details in the early stages of the design is expected to increase the quality of the results achieved by reducing the chance of costly redesign activities at a later stage.

The paper proposes a multilevel approach for the preliminary structural design process. The DLR in-house prototype tools for the parametric modeling and sizing of a wing finite element model (global level) are extended to cope with an equivalent model of the explicit stringer stiffened skin panels. Selected wing panels are extracted, and finite element models, which includes the explicit stringers components, are generated (local level). The global boundary conditions are transferred from the global to the local model, which is optimized under strength and additionally stability criteria. The optimal results are used to update the global model properties and a number of global-local optimizations cycles executed. A framework is developed to couple the level specific tools, and to manage the flow of variables among the two levels. The process targets the pre-design activities where frequent changes are necessary to explore the potential candidate solutions. The manipulation and the generation of finite element models are always time consuming activities, hence a parametric stiffened panel generator is implemented in order to reduce the repetitive operations. In order to improve the efficiency of the design process, ${ }^{2}$ the complete global-local cycle is designed to run fully automated with the minimum user intervention. Hence a set of additional routines are implemented serving the coordination of variables, the mapping between the levels' input-output, the management and the postprocessing of the results.

\section{Multilevel Analysis}

The multilevel methodology introduced is here described and applied for the preliminary structural design of transportation aircraft stiffened wings. The decomposition adopted proposes as global level the overall wing with the explicit modeling of the primary structures: skin, spars, and ribs. Whereas the wing substructures, such as wing stiffeners, are implicitly modeled ${ }^{3}$ by an equivalent layer formulation. The local level consists of extracting an arbitrary number of wing skin panels from the global level, which are remodeled with a higher number of substructural details. The global and the local levels modeling techniques are now introduced.

\section{A. Global Level Modeling}

The global wing structural level is represented by a multilayered shell finite element model, which is generated by using the prototype DLR application PARA_MAM. ${ }^{4}$ The fundamental principle of the automated modeling engine is to use the aerodynamic surface meshes available as contour reference for the generation of the Finite Element (FE) mesh, and to fit the inner structural components parametrical defined as input. The contour surface can be either an unstructured or a structured surface mesh used for high fidelity aerodynamic analysis, or can simply be a structured grid of points generated by the airfoil distribution in the span-wise direction. The tool PARA_MAM is realized as a set of MATLAB macros, and the wing configuration meshes are provided as ASCII files. Once the topology of the the structural lay-out (e.g. the ribs and spars relative positions and numbers) is parametrical specified, the code calculates the grid of points describing the ribs-spars intersections, which constitutes the primary definition of the wing structure. Hence as the structural grid mesh is calculated, all the structural elements (keypoints, lines and areas of spars, ribs, wing skin) are assembled in FE properties tables. The tool generates a collection of matrices containing the data required to define the model within the pre-processor of the FE package used. The structural properties are defined in the input file in a parametrical way as well, by specifying a default value and non-default ones for specific areas. Examples of properties specified are the thicknesses of the structural components, the material characteristics, and the orientation of the elements in the structure (e.g. when considering non isotropic materials). The code exports the final model and all the related properties into a set of input decks suitable for FE pre-processors operated in batch mode. The FE wing model output chosen is described by Ansys Parametric Design Language (APDL) format, which can be directly interpreted by the FE software package ANSYS, Inc. The wing mesh used as reference and the FE model generated are shown in figure 1. The explicit modeling of the stringers over the complete wing would dramatically increases the computationally cost for the preliminary structural design. Thus an implicit formulation is adopted to take into account the influence of the actual stringers elements in the global wing level. The multi-layered shell element type 


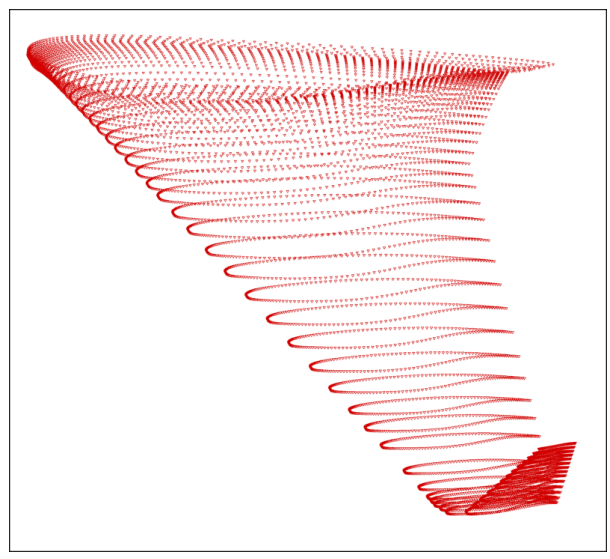

(a) Reference grid

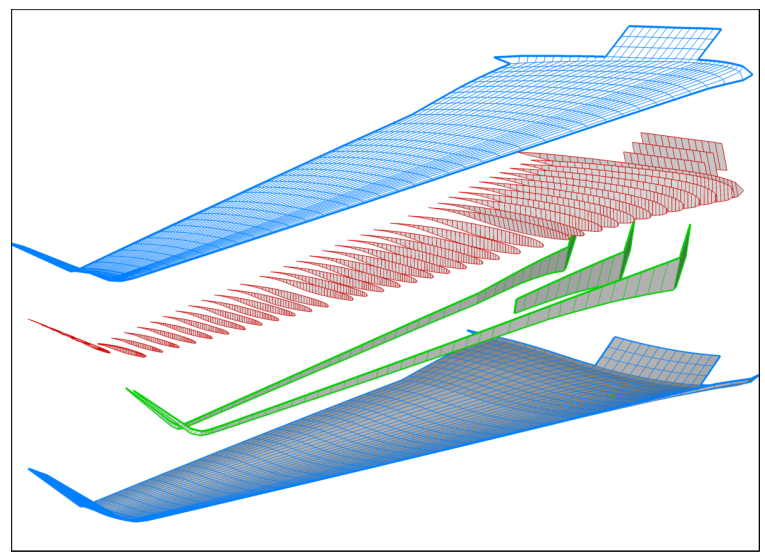

(b) Shell FE global model

Figure 1. Global level structural wing model.

chosen for FE global level supports the specification of the thermo-mechanical properties for each individual layer. Hence the stiffened wing skin panel are represented by an equivalent model defined by two type of layers: one representing the actual wing skin, and an equivalent layer defined by smearing the stringers properties.

As can be observed from figure 2, the first layer of the equivalent representation can be defined by simply specifying as real constants the properties of the actual skin layer (e.g. skin thickness t, material modulus $\mathrm{E}$ and density $\rho$ ). In the presented study the smearing approach is simplified by matching only the bending stiffness (EI), and the density of the actual stinger with the ones of the equivalent layer. Additionally the layered elements include in the set of allocated attributes the specification of the orientation for orthotropic material definitions. The equivalent layer is considered as an orthotropic homogeneous material, whose orientation is given by the orientation of the effective stringers. When the material used

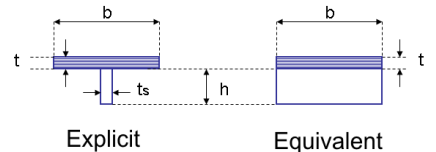

Figure 2. Explicit skin-stringer and Equivalent layered shell model for skin and stringers is isotropic and homogeneous only two layers of the shell elements are sufficient: the skin layer, and the equivalent layer. When composite laminates are used for the wing modeling, more layers are specified to reflect the number of laminae described by the stacking sequence of the skin panels, and the equivalent layer for the stiffener representation.

\section{B. Local Level Modeling}

The local level is an explicit model of the wing stiffened panel type selected, that is used to optimize the skin-stringer layout for additional failure modes, such as buckling failures, which cannot be included in the global formulation. A parametric panel engine generator is thus developed to automate the panel decomposition from the global level, and the remodeling with increased structural details. An arbitrary number of regions to be extracted from the global model is specified, and the corresponding wing geometry data are transferred to the panel generator, which creates the local panels with the skin-stiffener layout consistent with the implicit formulation. Similarly to the global level approach, the stiffened-panel generator consists of a set of MATLAB macros producing APDL input-decks for the bottom-up modeling and analysis of the panel in the FE package. The layered shell elements used can be employed to model both isotropic or anisotropic materials. The element structural properties (i.e., material characteristics, and layer thickness) are transferred from the selected region of the global model. A set of routines automates the process with the minimal user interaction. For the preliminary assessment of the whole multilevel process only blade stiffeners are considered in order to reduce the number of available design variables, although different design options could be modeled by using additional set of geometrical relations. The elastic FE analysis of the detailed panel under the applied boundary conditions provides the normal and shear stress state over the skin and the stringer elements. Figure 3 shows the decomposition approach for one panel extracted from the global 
wing model, and the mesh of the explicit stiffened-panel which is generated in the FEA ANSYS environment.

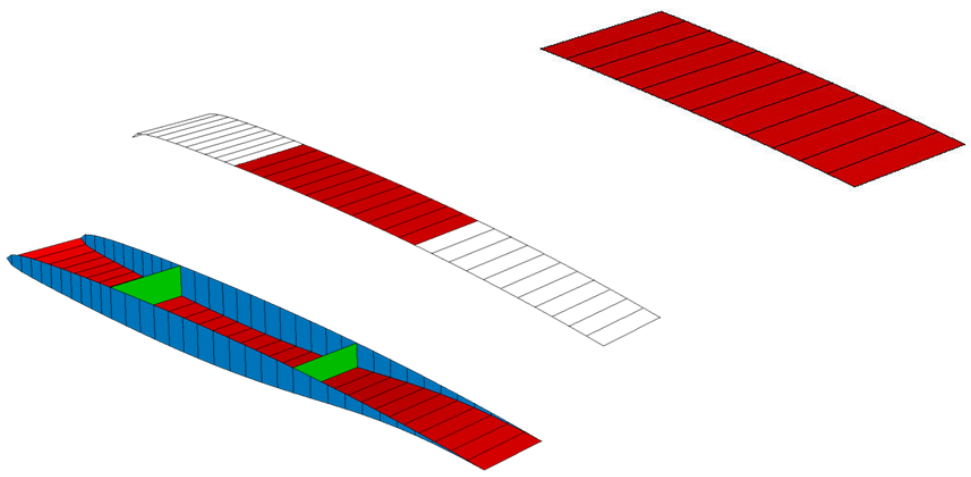

(a) Global model wing panel

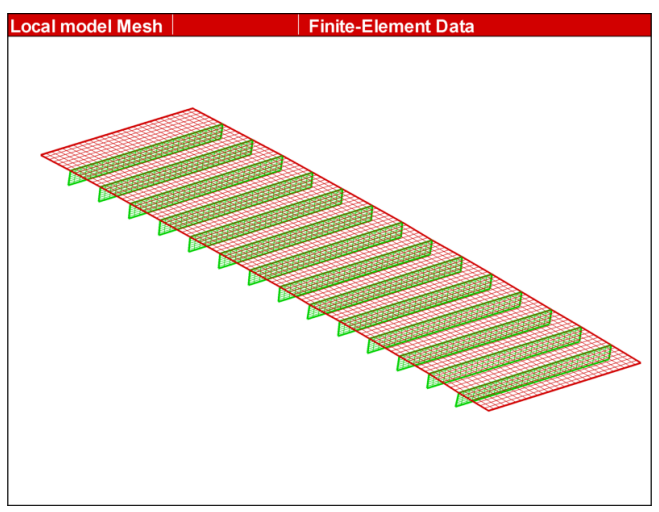

(b) Local model stiffened-panel FE mesh

Figure 3. Global Local Decomposition.

\section{Multilevel Optimization}

\section{A. Global Level Wing Sizing}

The global structural optimization aims to the minimization of the wing mass as objective function, under strength constraints and given aerodynamic loads. The bending and the torsional stiffness of the complete wing are assumed to mainly depend on the design of the wing box (i.e., the wing portion between the main front and rear spar), and not on the leading and trailing edges areas, where movables such as high lift devices and control surfaces are installed (e.g., flaps, slats, ailerons). Thus the structural optimization problem involves only the wing box elements, and can be formulated as:

$$
\text { Global Sizing }\left\{\begin{array}{l}
\text { given the applied loads } \\
\text { minimize the wing box components mass (Objective function) } \\
\text { by varying the wing box elements thicknesses (Design variables) } \\
\text { subject to the failure criteria specified (Design constraints) }
\end{array}\right.
$$

The design variables are defined for each element of the wing components in terms of layers' thicknesses and orientation angles for non isotropic material. The amount of design variables for a typical problem can range from $50 \mathrm{k}$ to $5 \mathrm{M}$, and finding the best combination for all of them cannot be directly handled by the mathematical optimization techniques. The fully stress based sizing infrastructure S_BOT (Sizing roBOT) developed by DLR is used to determine the global wing model thickness distribution. The tool's main input file calls the FE software, reads the global FE model generated, applies the specified loads (e.g. the aerodynamic pressure distribution), launches the analysis solver, and post-process the loading state for 
each element of the structural wing box components. Thus on the base of the sizing criterium specified in the tool, the new elements dimensions are calculated, and the thicknesses of the skin element layers are updated for all wing elements. After the model updating the load path is changed, and the analysis-sizing process is iteratively repeated till convergence criteria are met. For the isotropic material design case in this paper the maximum stress criteria is selected for the elementwise sizing of the global level. Figure 4 shows the typical thickness distribution on the upper skin of the wing box elements for different iterations. The structural components mass is monitored at each iteration as shown in figure 5(a), and the wing deflection is tracked on the wing tip node as shown in figure $5(\mathrm{~b})$.

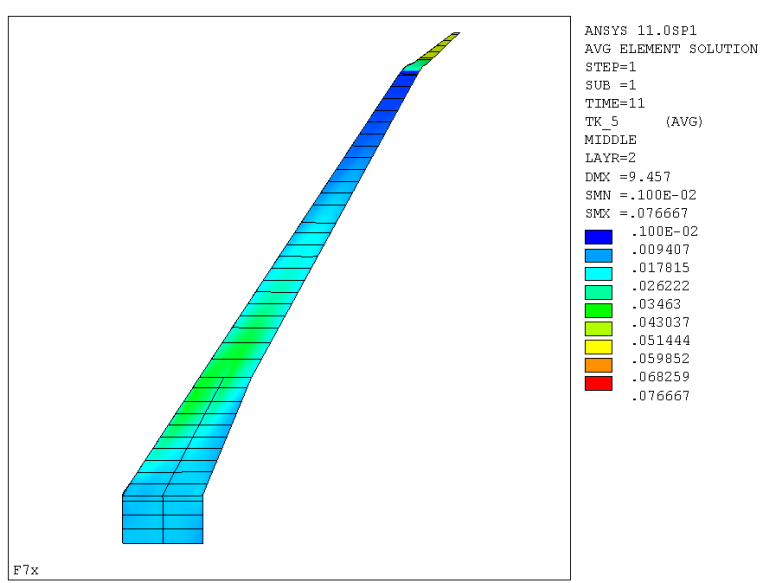

(a) Thickness Iteration 5

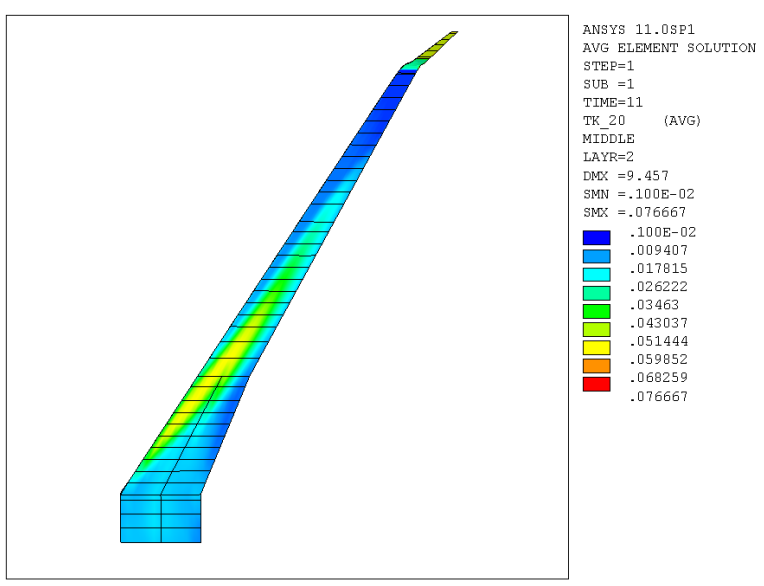

(b) Thickness Iteration 20

Figure 4. Element thickness distribution wing box

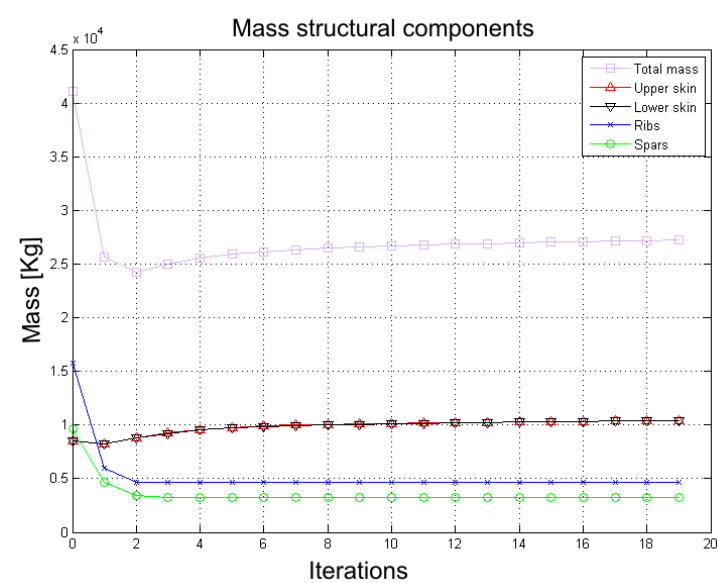

(a) Mass structural components

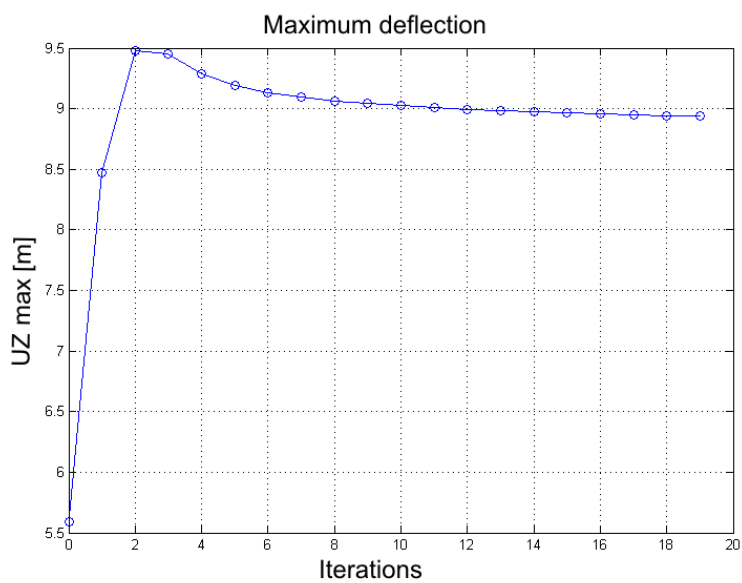

(b) Wing tip deflection

Figure 5. S_BOT sizing routine monitoring

\section{B. Local Level Panel Analysis}

Once the FE stiffened panels models are generated, a module provides the nodal mapping between the global and the local levels. From the global wing FEA solution, stress and displacement fields are generated and used as load cases for the local panel analysis. Figure 6 shows for instance the global nodal displacements in the $\mathrm{z}$ direction for a selected wing panel, and the corresponding displacement field generated, and distributed over the local model nodes. 


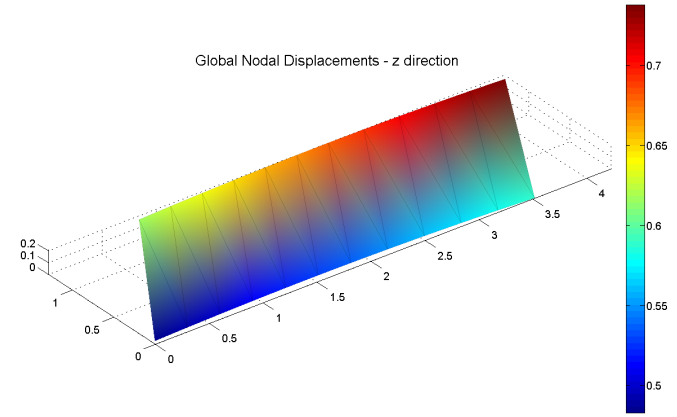

(a) Global panel nodal displacements [m]

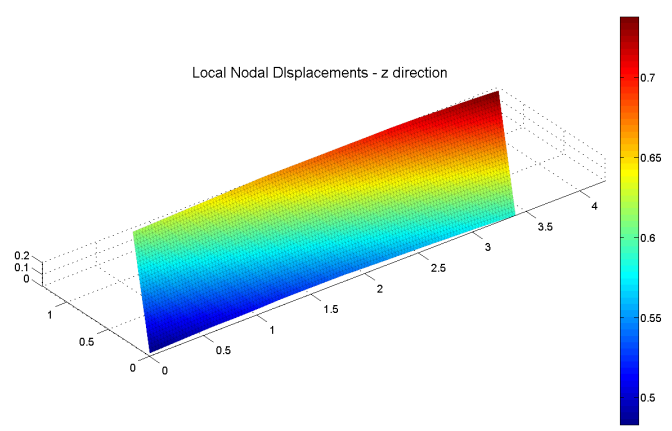

(b) Local panel nodal displacements [m]

Figure 6. Nodal Mapping module

Once the boundary conditions are transferred from the global to the local model, the stress state on the local stiffened panel model is analyzed. Thus a local optimization is set up to design the skin-stringer layout for minimum mass, against strength and initial stability failure criteria. Several instability modes of panels are included and predicted by following the instability of plates theory, engineering methodologies and design rules provided for instance by the ESDU (Engineering Sciences Data Unit). Although the panel generator produces a detailed FE model of the curved stiffened-panel, for the prediction of the critical loads a non curved analytic approximation is introduced in this work, in order to assess the overall approach. The following geometry notations are introduced:

- $\mathrm{t}$ : the wing skin thickness of the panel

- b: the stringer pitch

- $t_{s}$ : the blade stringer thickness

- h: the stringer height

- L: Panel length (rib pitch)

The flexural buckling is taken into account by considering a stringer and the portion of adjacent skin as a simple strut between ribs. The panel behaves as an Euler column ${ }^{5}$ and the critical stress at which the panel initially buckles is expressed by the formula 1 :

$$
\sigma_{F}=K_{F} E_{g}\left(\frac{b}{L}\right)^{2}
$$

where the flexural buckling coefficient $K_{F}$ depends on the given shape of the panel. The local buckling mode depends on the geometry of the skin-stringer combination, and the stress level at which the plate starts to buckle is expressed by the Eq. 2 .

$$
\sigma_{L}=K_{L} E\left(\frac{t}{b}\right)^{2}
$$

The local buckling coefficient $K_{L}$ is made available through design curves, ${ }^{6}$ given the panels shape ratios $t_{s} / t$ and $h / b$. Further for isotropic materials a module is implemented to take into account the plasticity effect of the deformation by including the tangent modulus $E_{t}$ through an iterative procedure. The shear stress is derived from the FE analysis of the panel, and the shear buckling load of the panel is included as well. This can be expressed as:

$$
\tau_{c r}=K_{s h r} E\left(\frac{t}{L}\right)^{2}
$$


where an approximated expression is used for coefficient $K_{s h r}{ }^{7}$ The FE analysis module provides the stress state over the stringer elements as well. Thus a local stringer buckling criterium is added. The blade stringer is considered as a simple-supported plate with one edge free, and for isotropic material the critical load is given by: ${ }^{8}$

$$
\sigma_{L S}=0.385 E\left(\frac{t s}{h}\right)^{2}
$$

\section{Local Level Panel Optimization}

Once the stiffened-panel failure behavior is modeled the local optimization problem can be formulated introducing the design variable vector $\mathbf{x}$, an objective function $\mathrm{f}(\mathbf{x})$, a set of equality and inequality constraints $h_{k}(\mathbf{x})$ and $g_{j}(\mathbf{x})$ defining the design space, and a set of upper and lower bounds on the design variables. The optimization problem is mathematically defined as: ${ }^{9}$

$$
\begin{array}{lll}
\text { minimize: } & f(\mathbf{x}) \\
\text { by varying: } & \mathbf{x} \in \mathbb{R}^{n} & \\
\text { subject to: } & h_{k}(\mathbf{x})=0 ; \quad \text { with } \mathrm{k}: 1, \ldots, m \\
& g_{j}(\mathbf{x}) \geq 0 ; \quad \text { with } \mathrm{j}: 1, \ldots, l \\
& \text { lower bounds }<\mathbf{x}<\text { upper bounds }
\end{array}
$$

The blade stiffened-panel is locally optimized for minimum weight, thus the panel mass per unit area is set up as objective function and it is expressed by the following equation:

$$
f=\rho\left(t+\left((b(h-t / 2)) / t_{s}\right)\right) / 1000 ; \quad\left[K g / m^{2}\right]
$$

where the design variables considered are the skin-stringer combination dimensions: $\mathrm{t}, \mathrm{b}, t_{s}, \mathrm{~h}$.

The theoretical optimum design of a stiffened-panel is obtained for a simultaneous mode design (optimality criteria) ${ }^{10}$ which can be expressed as:

$$
\sigma_{F}=\sigma_{L}
$$

However in practical problems the maximum efficiency corresponds to a low value of stringer spacing, which affects the weight of the panel. Thus the stringer pitch variable is lower bounded for a minimum value $b_{\min }$. The following inequality constraints functions are included to evaluate the material strength and the panel stability, and to upper and lower bound the design variables.

$$
\left.\begin{array}{l}
\sigma<\sigma_{\text {all }} \\
\sigma<\sigma_{F} \\
\left(\sigma / \sigma_{L}\right)+\left(\tau / \tau_{c r}\right)^{2}<1 \\
b>b_{\min } \\
h<h_{\max } \\
h<b \\
h>0.2 b \\
t_{s}<3 t \\
t_{s}>0.8 t
\end{array}\right\} \text { Inequality constraints }
$$

The local panel optimization is implemented in MATLAB as a nonlinear constrained minimization problem, by using the Sequential Quadratic Programming (SQP) as solution algorithm. The design parameters, such as the panel dimension, are determined by the spanwise location of the panel in the global model; as well as the panel load intensity, which is calculated during the FE local analysis, and given as input for the panel optimization. The local panel optimization module is here tested for a range of the panel length $\mathrm{L}$ and normal compression load intensity p. The parameters boundary are swept by the values in table 1 . The design space is then mapped by a full factorial Design Of Experiment (DOE) resulting in 225 optimizations. Each experiment point in figure 7 is a mass optimized panel design for the corresponding values of $\mathrm{p}$ and 
L. The DOE of the local level may be repeated for other types of local structural concepts, and the results used for the construction of response surfaces and the comparison of the different structural solutions. ${ }^{11}$

\begin{tabular}{lcc}
\hline Parameter & Lower bound & Upper Bound \\
\hline $\mathrm{L}[\mathrm{mm}]$ & 50 & 2000 \\
$\mathrm{p}[\mathrm{N} / \mathrm{mm}]$ & 500 & 8000 \\
\hline
\end{tabular}

Table 1. DOE boundaries for the local optimization

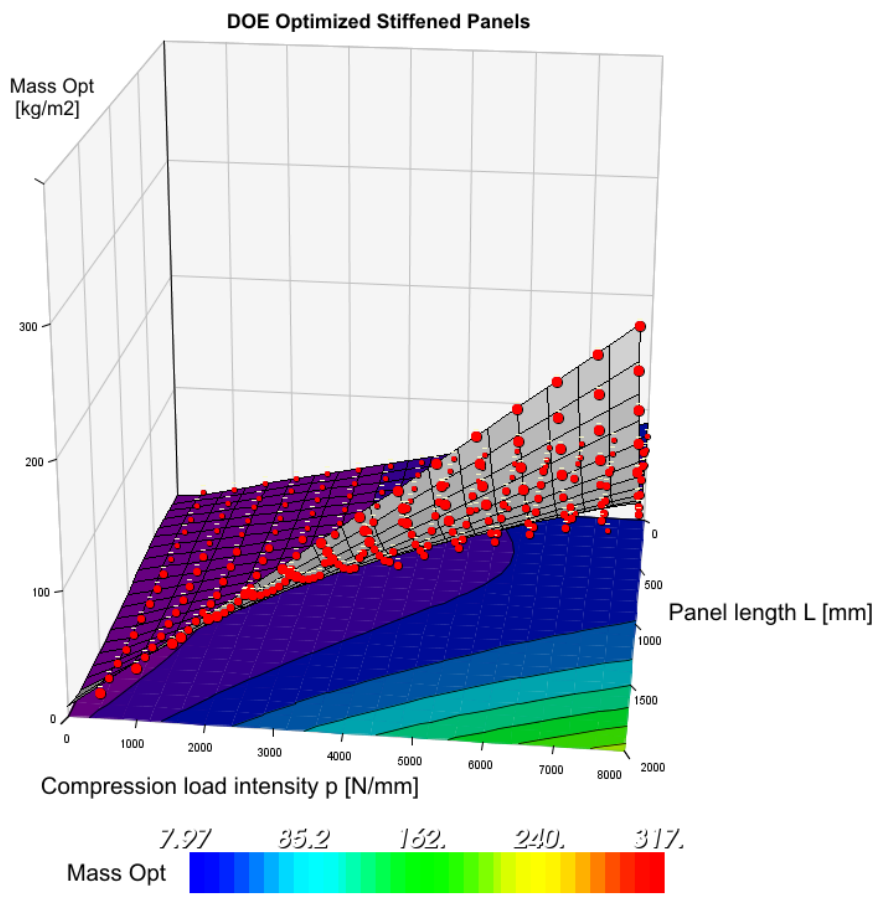

Figure 7. DOE Experiments optimized panel Mass

\section{Global Local Integration Strategy}

A framework is implemented to serve the communication among the global and the local levels. The first step is the calculation of the wing geometry. The FE wing model includes the equivalent structural properties derived from the initially specified skin-stinger layout. The FE global analysis module is then called to analyze the wing model under the load cases provided. In this phase the structural sizing routine minimizes the wing box mass subjected to the global optimization constraints, and the optimal thickness distribution over the wing box elements is obtained. Once the global sizing converges, the last iteration thickness distribution is transferred to the panel generator which produces the FE models of the detailed stiffened panels selected. Thus the global nodal boundary conditions are extracted from the wing FEA results and mapped over the FE panels models nodes. Subsequently a FE analysis is performed, and the resulting loading states over the stiffened-panels components are used as input for the local optimization. The local optimization routine simplifies the curved and skewed FE model with a flat and rectangular stiffenedpanel representation subjected to in-plane loading for the calculation of the critical initial buckling failure modes, and optimizes the stringer-skin dimensions for minimum weight under the strength and the stability constraints which are non considered in the global sizing. The optimal sized layout is transferred back to the global model which is updated with the new properties, and can be globally sized again. A number of 
iterative global-local sizing cycles are established, and the structural components masses are monitored for the global sizing, the local optimizations, and the overall global-local approach. The figure 8 shows the flow chart of the process described.

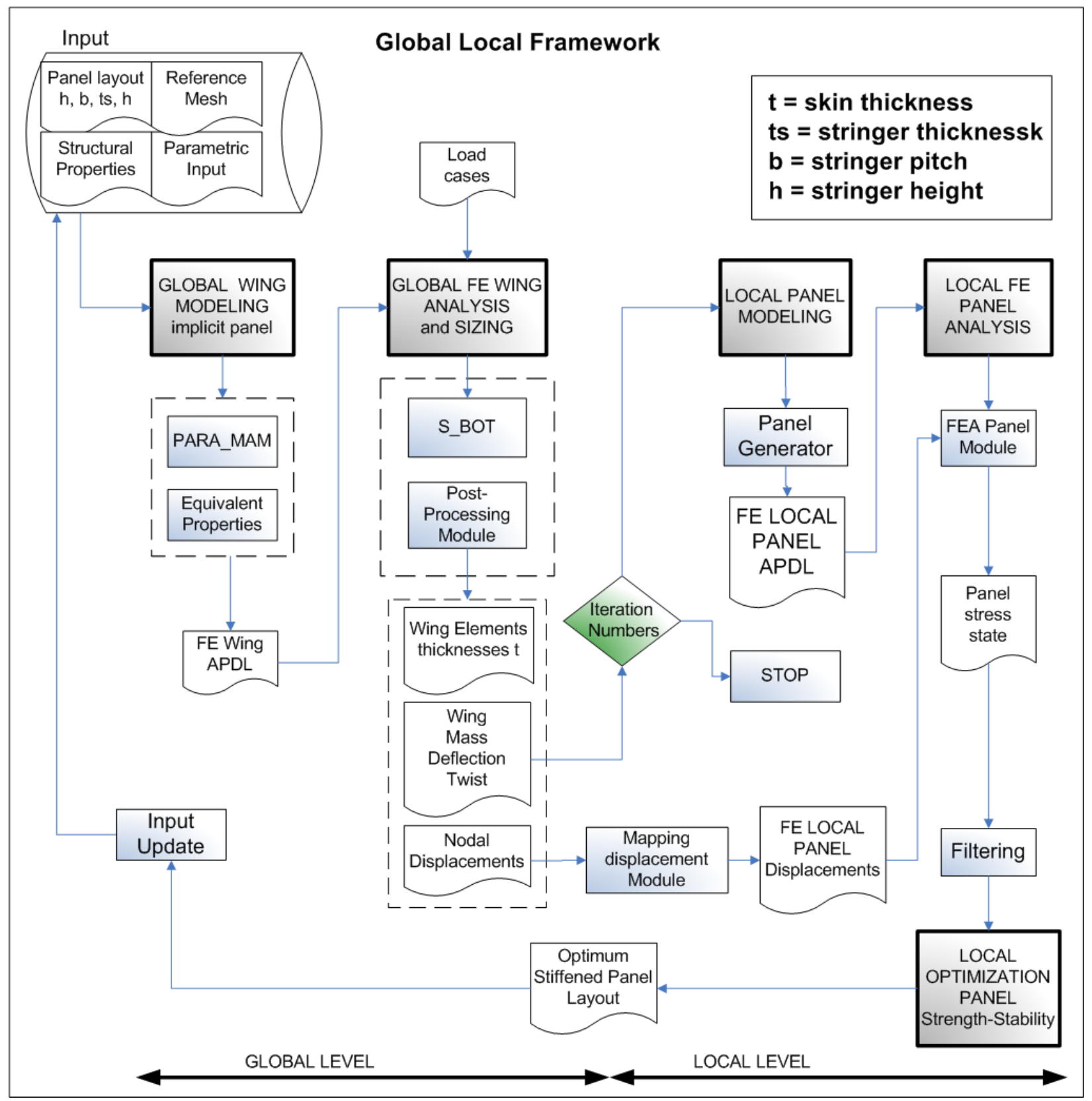

Figure 8. Global-Local Integration Strategy

\section{A. Global Local Design Case}

The global local optimization approach is tested for the multilevel structural sizing of a DLR designed wing for large transportation aircraft. In the main input an arbitrary number of wing panels can be selected to be used for the local optimization, and their optimal results are used to update all the skin elements properties over the complete global wing panels. As a limit case all the global wing surface can be spanned and each of the single panel further remodeled, analyzed and locally optimized. In order to track the process 
behavior only three representative panels are selected to be explicitly modeled in this paper study, which are respectively positioned in the area near the wing root, the mid wing section and the wing tip. The three panels chosen are positioned between the two main wing spars, and the two adjacent ribs at different span wise stations as shown figure 9 .

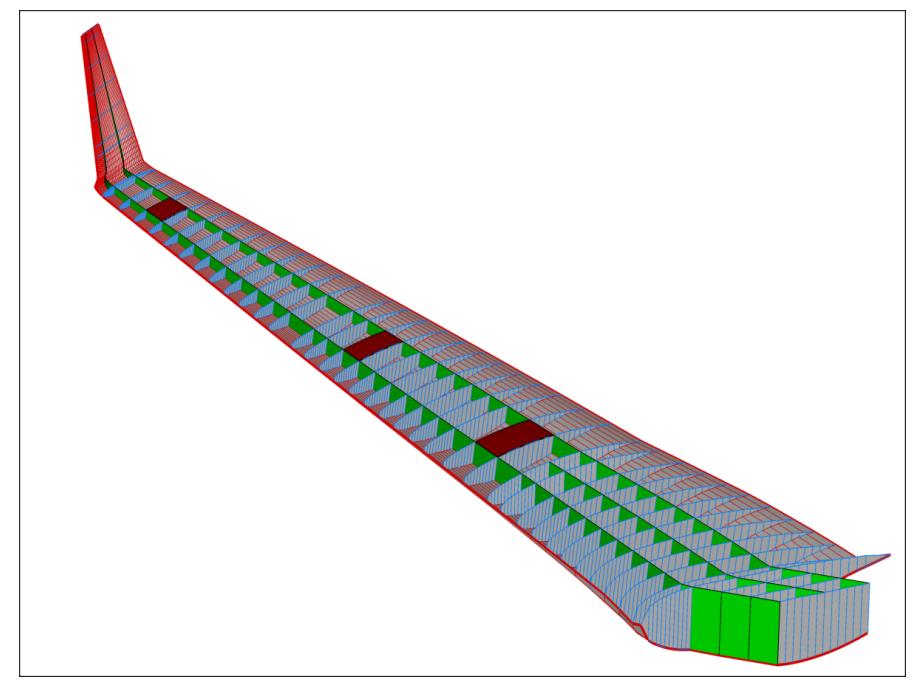

Figure 9. 3 Panels selected for the local optimization

\section{Results}

The global-local optimization results are presented for the multilevel structural sizing of the tested wing. As described in the previous section the multilevel automated procedure is tested by performing the local optimization over three explicitly modeled panels. The loading condition selected for the design case is a $2.5 \mathrm{~g}$ manoeuvre, which produces the highest compression loads on the upper wing skin elements. For the preliminary assessment of the approach the test wing is modeled by using isotropic materials whose mechanical properties are reported in table 2.

\begin{tabular}{lcccccc}
\hline Material & $\mathrm{E}[\mathrm{MPa}]$ & $\nu$ & $\mathrm{G}[\mathrm{MPa}]$ & $\rho\left[\mathrm{Kg} / \mathrm{m}^{3}\right]$ & $\sigma_{\text {all }}[\mathrm{MPa}]$ & $\mathrm{m}$ \\
\hline $\mathrm{Al} 7075 \mathrm{~T} 6$ & 71700 & 0.33 & 26900 & 2800 & 470 & 14 \\
$\mathrm{Al} \mathrm{2024}$ & 73100 & 0.33 & 27600 & 2800 & 360 & 14 \\
\hline
\end{tabular}

Table 2. Material properties specified

The material strength allowable $\sigma_{\text {all }}$ effectively used in the sizing modules is reduced by a safety factor, whereas the factor $\mathrm{m}$ characterizes the stress-strain curve to account the plasticity effect of the deformations. The initializing dimensions specified for the initial stiffened panel layout, and the other structural components (e.g., spars and ribs thickness elements) follows:

$$
\begin{aligned}
& t_{0}=30[\mathrm{~mm}] \text { (Wing skin thickness) } \\
& b=200[\mathrm{~mm}] \text { (Stringer pitch) } \\
& t_{s}=40[\mathrm{~mm}] \text { (Stiffener thickness) } \\
& h=50[\mathrm{~mm}] \text { (Stiffener height) } \\
& t_{s p}=100[\mathrm{~mm}] \text { (Spars elements thickness) } \\
& t_{r i}=100[\mathrm{~mm}] \text { (Ribs elements thickness) }
\end{aligned}
$$

Thus the global wing model is generated by PARA_MAM, and globally sized by the S_BOT module which 
provides the initial thickness distribution. The evolution of the skin thickness with the global iterations is shown in figure 10, where a filter has been applied to the elementwise values in order to reduce the thickness discontinuities.

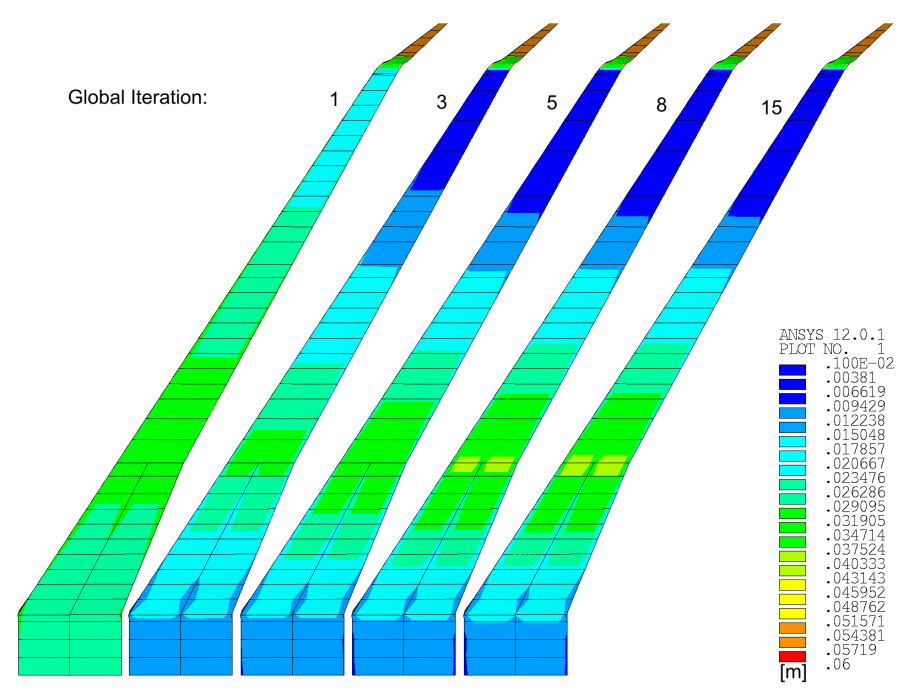

Figure 10. Initial global optimization thickness distribution

Thus after the initial global sizing the thickness distribution is transferred to the local panel generator module, and the global displacement are mapped on the local detailed panels selected. Hence the results of the local analysis are post-processed and filtered to derive the stress state of the stiffened panels components, which is transferred to the local optimization module.

In figure 11 both the FE global and local models are plotted together in their global reference system coordinates used for the analysis. The figure 11(a) shows the consistency between the two models (only shown for the mid section panel) either for the deformed and undeformed conditions. A closer detail of the superposition of the two FE models is in figure 11(b).

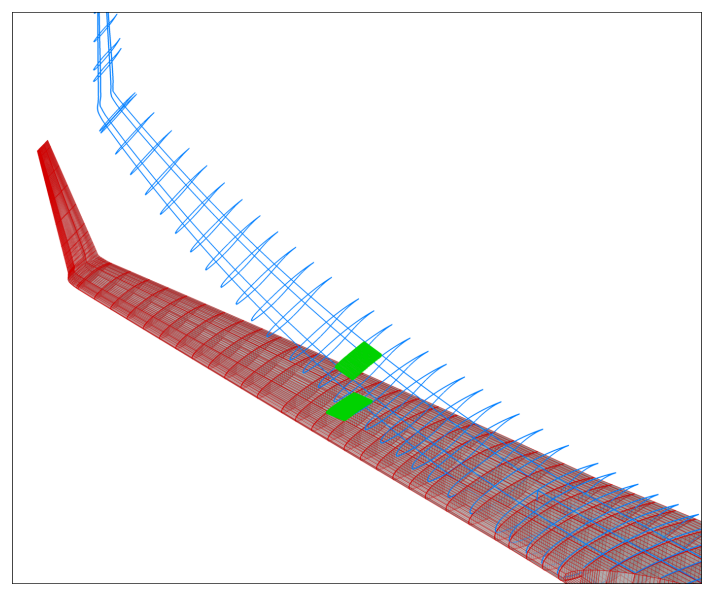

(a) FE models deformed-undeformed

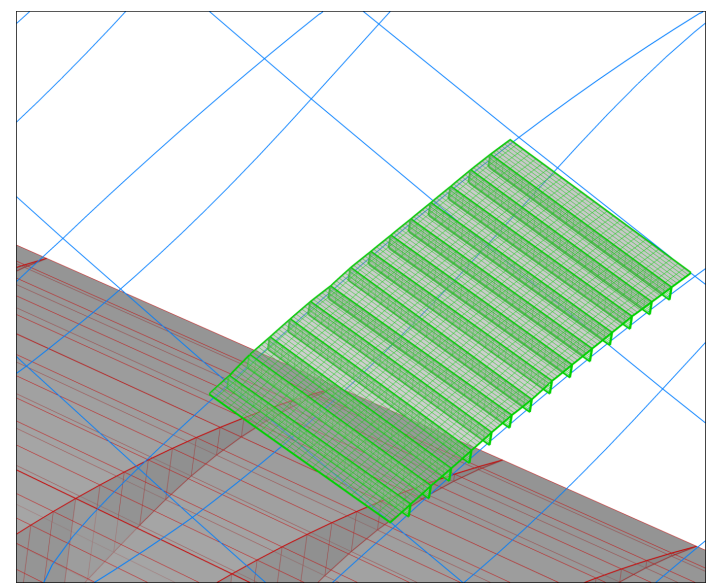

(b) Models Detail

Figure 11. Global-Local models post-processing consistency

Table 3 specifies the three panel properties before the local optimization. The thickness $t$ is the one derived from the last iteration of the global sizing. The axial normal load intensity $\mathrm{p}$ is extracted from the FE analysis of the local panels. Other panels properties such as the length $\mathrm{L}$ and the width $\mathrm{W}$, and the initial panels mass per unit area are included as well. 


\begin{tabular}{lccccc}
\hline Panel ID & $\mathrm{t}[\mathrm{mm}]$ & $\mathrm{p}[\mathrm{N} / \mathrm{mm}]$ & $\mathrm{L}[\mathrm{mm}]$ & $\mathrm{W}[\mathrm{mm}]$ & $M_{\text {init }}\left[\mathrm{Kg} / \mathrm{m}^{2}\right]$ \\
\hline Root & 48 & 3400 & 1300 & 3800 & 148.9 \\
\hline Mid & 36 & 2915 & 900 & 3000 & 118.1 \\
\hline Tip & 9 & 2790 & 900 & 1700 & 50.3 \\
\hline
\end{tabular}

Table 3. Local panels initial properties

The panels properties are transferred to the local optimization module, together with the main input data. The initial design variable vector is defined as:

$$
\begin{aligned}
\mathbf{x}_{0} & =\left[t, b, t_{s}, h\right] \\
\text { with } b_{\min } & =100[\mathrm{~mm}] \\
\text { and } h_{\max } & =80[\mathrm{~mm}]
\end{aligned}
$$

The new dimensions, the optimized panel mass $M_{o p t}$, the local and flexural buckling coefficients calculated are reported in table 4. Figure 12 shows the design variables, and the target function during the first local optimization for one of the panels optimized. It can be observed the large mass reduction, due to the initial non optimum stiffened panel layout.

\begin{tabular}{lccccccc}
\hline Panel ID & $\mathrm{t}[\mathrm{mm}]$ & $\mathrm{b}[\mathrm{mm}]$ & $t_{s}[\mathrm{~mm}]$ & $\mathrm{h}[\mathrm{mm}]$ & $M_{\text {opt }}\left[\mathrm{Kg} / \mathrm{m}^{2}\right]$ & $K_{L}$ & $K_{F}$ \\
\hline Root & 6.5 & 201 & 19 & 74 & 37.6 & 5.9 & 0.13 \\
\hline Mid & 6 & 201 & 18 & 53 & 30.0 & 6.2 & 0.087 \\
\hline Tip & 7 & 202 & 14 & 61 & 29.4 & 6.09 & 0.082 \\
\hline
\end{tabular}

Table 4. Optimum local panels designs

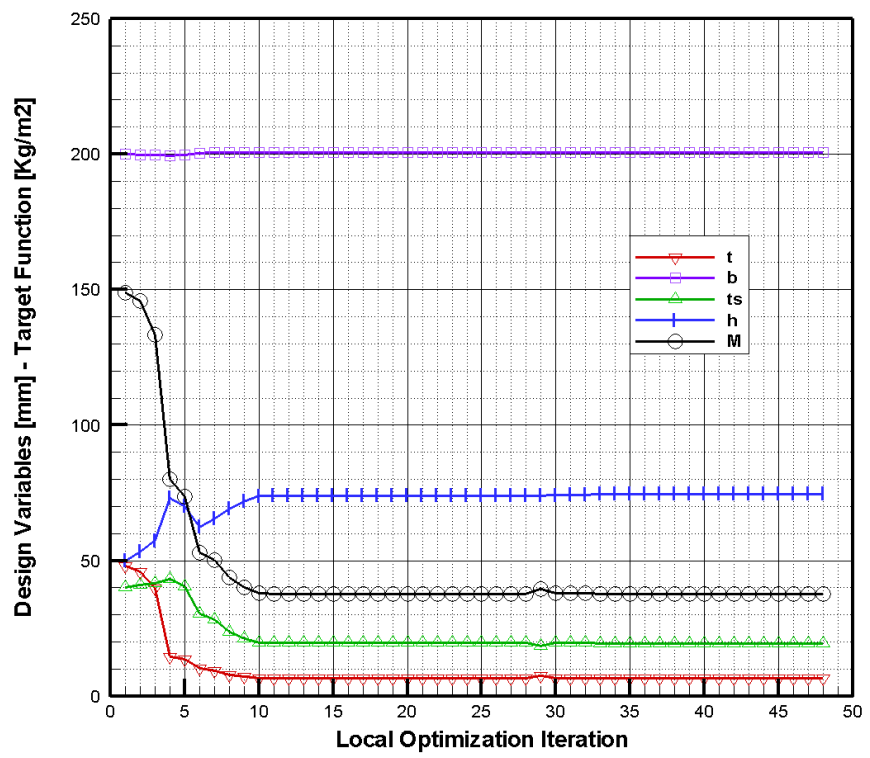

Figure 12. Local Optimization for the Root Panel 
The overall global model is then updated with the optimal values from the local optimizations, and the complete global-local cycle runs for a number of iteration. The next subsection presents the results calculated for a maximum number of 15 global-local loops.

\section{A. Global-Local Mass Comparison}

The complete global-local cycle is repeated till convergence criteria are satisfied (i.e. maximum number of iterations, or tolerance on the mass values). Thus the initial global sizing, subjected to only the strength criteria, is compared with the global sizing relative to the last global-local cycle, in which the equivalent layers properties are calculated on the basis of the local optimizations, which include the buckling failure modes. The overall optimization is run either by making use of the three explicit local panels in the design case, either by increasing the number of panels to six for the local optimizations. The number of global-local cycles is investigated till a maximum of 15 loops. Figure 13 shows the comparison of the only global sizing approach (initial global sizing), and the global sizing results from the last global-local loop with for different number of local optimizations involved. In figure 13(a) the plotted lines indicate the sum of the element masses relative to the complete wing box (ribs, spars, upper and lower skin implicit panels), whereas in figure 13(b) only the sum of the elements masses relative to the upper skin, which is the component more affected by compression loads, hence to stability failures. The results are normalized with the value of the wingbox mass at the initial the global-local approach.

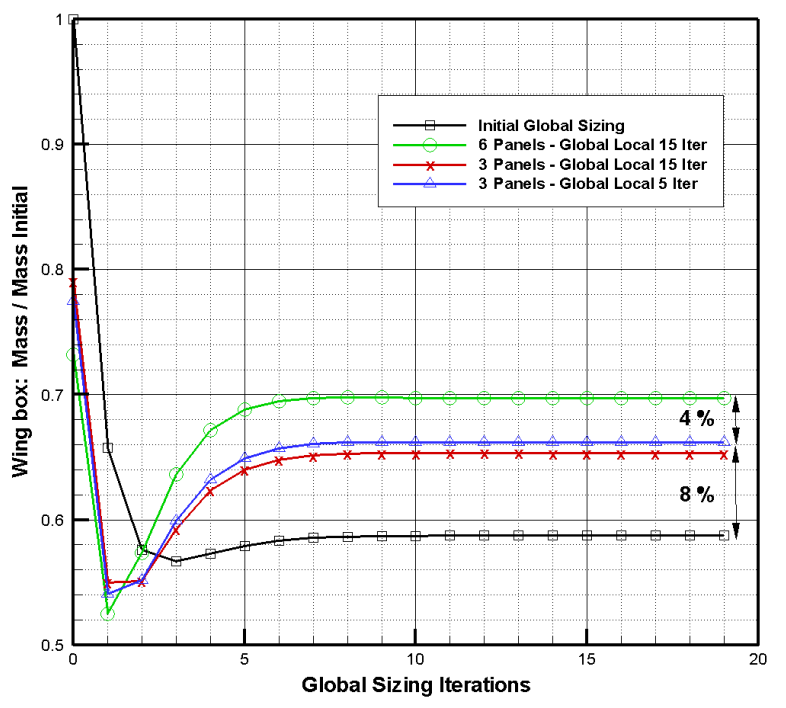

(a) Wing box elements mass

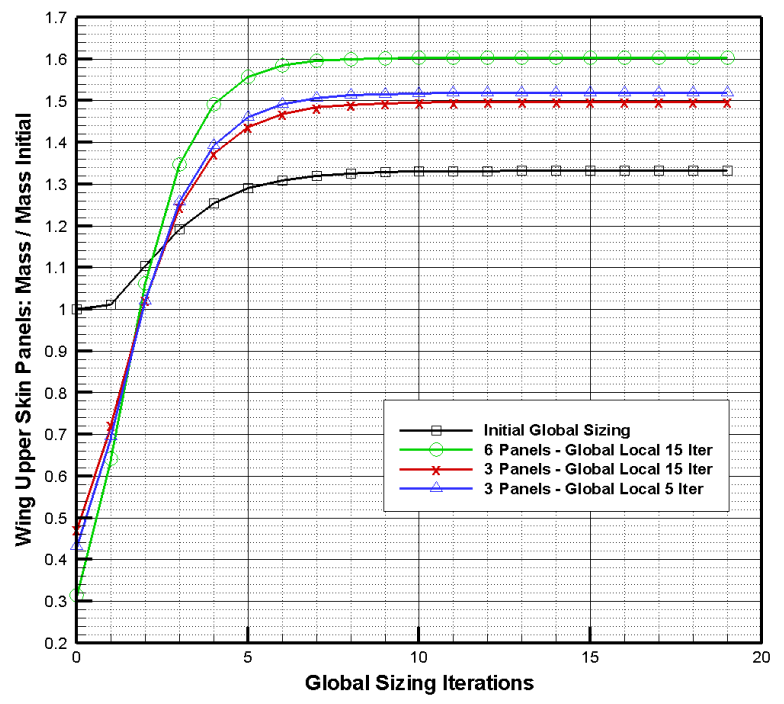

(b) Upper skin panel elements mass

Figure 13. Global sizing vs Global-Local approach

The global-local optimized structures provides a final design with a $8 \%$ mass increase respect to the only global fully stressed design, which it is expected since additional failure modes are included. Increasing the number of panels a further difference can be observed, since detailed analysis are performed at more wing stations. The number of the overall global-local cycles does not show a substantial difference in the final results. The total wing box, and the upper skin panels masses resulting from each global-local cycle are shown in figure 14, both for the case employing three and six local panels. It is observed that the multilevel approach can provide stable results with an already low number of loops, whereas the number of local panels has a higher impact. The local optimizations performed at each global-local cycle are shown in figure 15 for the panel located at the inner section of the wing. Either the design variables (stiffened panel layout), either the target function (mass per unit area) evaluated at the design optima are plotted. Even in this case the multilevel optimization shows a stable behavior after few global-local cycles. 


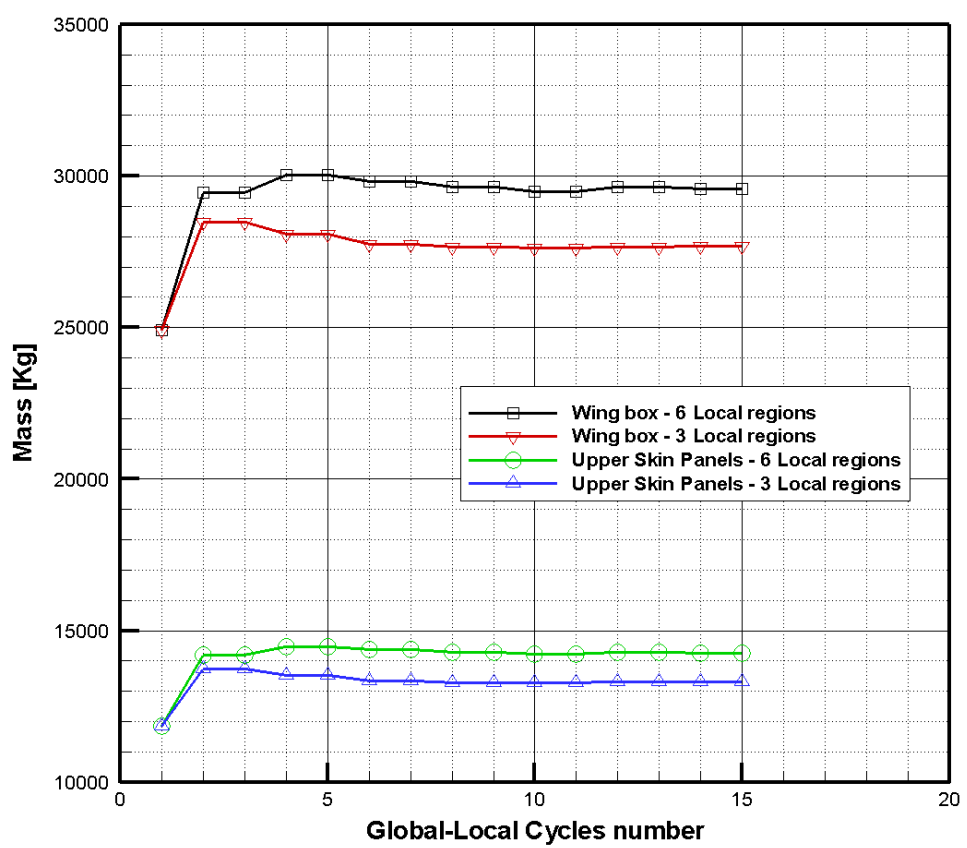

Figure 14. Global-Local Cycles-Wing Elements Masses

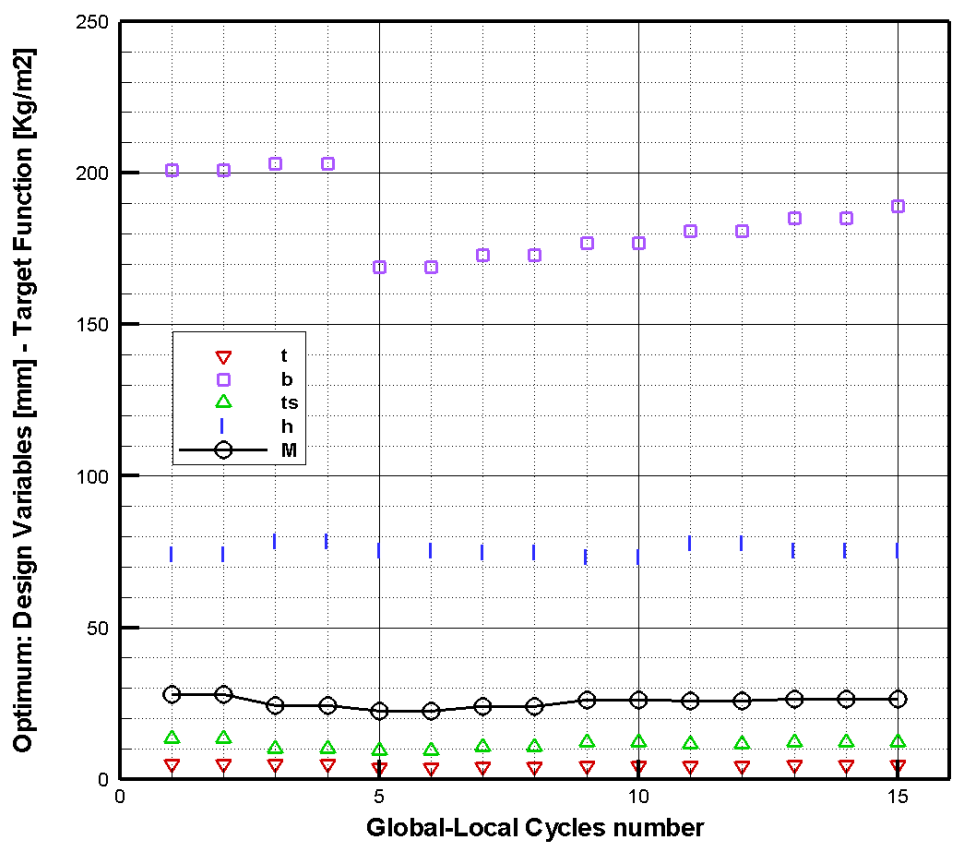

Figure 15. Local Optimizations: Global-Local Cycles-Optimum panel layout 


\section{B. Global-Local Computational Time}

The computational time breakdown of the complete process for the design case is indicated for one globallocal cycle in table 5. The most time consuming activity is the FE analysis and sizing of the main wing, depending on the number of global iterations. The FE modeling and analysis of the local explicit panels add only a limited amount of time, thus a higher number of local panels could be adopted. Whereas the time required by the local optimization module is much more lower, although the MATLAB algorithm did not always provided a convergent solution. The updating. The mapping module, and the the updating process are not expensive as well. The first phase runs only for the first cycle, since the updating process is based on changing the shell element properties, and not on the complete regeneration of the model. As shown in the previous section a low number of cycles is sufficient.

\begin{tabular}{lc}
\hline Phase & Time [s] \\
\hline Global Wing Modeling & 240 \\
Wing FE analysis and sizing (20 iterations) & 680 \\
Local Panels FE modeling and analysis (3 panels) & 160 \\
Global Local Mapping & 120 \\
Local Optimization (3 panels) & 60 \\
Updating & 40 \\
\hline
\end{tabular}

Table 5. Computational time global-local cycle

\section{Conclusions}

A global-local optimization methodology is presented for the structural design of transportation aircraft wings. The wing optimization problem is decomposed into a global wing and local panels subproblems. This methodology enhances the preliminary design stage by integrating structural details, and failure modes which conventionally would require computationally expensive fully detailed models. Variable fidelity analysis codes, and tools are integrated into a framework, which is design to automate the complete process. The methodology is applied for the design case of an isotropic wing structures, making use of blade stiffened panels. The potentialities of the multilevel optimization are investigated, and the benefit are shown to be achievable after a low number of global-local cycles. The results monitored are consistent with the level of the assumptions made.

\section{References}

${ }^{1}$ Ragon, S. A., G. Z. H. R. T. and Tzong, T. J., "Bilevel Design of a Wing Structure Using Response Surfaces," Journal of Aircraft, Vol. 40, No. 5, 2003.

${ }^{2}$ La Rocca, G. and van Tooren, M. J. L., "Enabling Distributed Multidisciplinary Design of Complex Products: A Knowledge Based Engineering Approach," Journal of Design Research, Vol. 5, No. 3, 2007, pp. 333-3352.

${ }^{3}$ Lisandrin, P. and van Tooren, M., "High-order finite elements reduced models for modal analysis of stiffened panels," Int.J.Mech.Mater.Des, Vol. 3, 2006, pp. 111-127.

${ }^{4}$ Nagel, B., Rode, M., and Monner, H., "An alternative procedure for FE-wing modelling," DGLR, , No. $048,2006$.

${ }^{5}$ Timoshenko, P. S. and Gere, M. J., Theory of elastic stability, McGraw-Hill, New York, NY, 1988.

${ }^{6} \mathrm{ESDU}$, "Information on the use of data items on the buckling of plates and compression panels manufactures from isotropic materials," 1972.

${ }^{7}$ Rothwell, A., "Structural Design and Optimization," Delft ae4-535 course.

${ }^{8}$ Megson, H. G. T., Aircraft structures for engineering students, Elsevier, 1999.

${ }^{9}$ Vanderplaats, G. N., Multidiscipline Design Optimization, Vanderplaats Research \& Development, Inc., 2007.

${ }^{10} \mathrm{Niu}, \mathrm{C} .-\mathrm{Y} . \mathrm{M} .$, Airframe stress analysis and sizing, Conmilit Press, 1997.

${ }^{11}$ Schut, E. and Van Tooren, M. J. L., "A Knowledge Based Engineering approach to automation of conceptual design option selection," 45th AIAA Aerospace Sciences Meeting and Exhibit, AIAA Paper 2007-968, Reno, Nevada, 2007. 